\title{
States of Ignorance and Ignorance of States: Examining the Quantum Principal Principle
}

\author{
Alexander Meehan*
}

February 1, 2021

\begin{abstract}
Earman (2018) has recently argued that the Principal Principle, a principle of rationality connecting objective chance and credence, is a theorem of quantum probability theory. This paper critiques Earman's argument, while also offering a positive proposal for how to understand the status of the Principal Principle in quantum probability theory.
\end{abstract}

\section{Introduction}

A particle is approaching a Stern-Gerlach magnet oriented in the $x$ direction. You know the particle is in the spin-up- $z$ state, $\uparrow z$. How confident should you be that an 'UP' outcome will be registered? Answer: you should be $50 \%$ confident. Here's one story for why. Knowing the particle is in the $\uparrow_{z}$ state tells you that the chance of ' $U P$ ' is $\left|\left\langle\uparrow_{x} \mid \uparrow_{z}\right\rangle\right|^{2}=1 / 2$. And epistemic rationality demands you conform your credences to known chances.

The last step in this reasoning is an application of the Principal Principle, which says (roughly) that if an agent knows the objective chance of a proposition $E$ is $x$, then she should set her degree of belief in $E$ to $x$ (Lewis, 1980). It is controversial what quantum chances really are, and if they are objective. But if they are objective, then this looks like a paradigm application of the Principal Principle.

On the standard way of understanding the Principal Principle, it is a normative requirement that goes above and beyond other more background constraints on rational credence, like proba-

*Department of Philosophy, Princeton University. Email: alexandermeehan@princeton.edu. 
bilism and conditionalization. While authors have disagreed about whether the principle can be independently justified and if so how (Strevens, 1999; Ward, 2005; Pettigrew, 2012, 2013; Childers, 2012; Schwarz, 2014; Builes and Spencer, ms), almost all agree that an agent can be probabilistically coherent and yet still violate it. However, Earman (2018) has recently suggested that in the quantum context, the principle:

Quantum Principal Principle (QPP), rough version: if an agent learns that the state of the system is $\psi$, then she should set her credence in $E$ to $\psi(E)$, the chance $\psi$ assigns to $E$,

follows as a "theorem of quantum probability theory", together with the usual coherence requirements on rational credence. Thus, there is no need to seek a further justification of the principle in the quantum setting, or to agonize over which variant of it is correct.

This paper assesses Earman's proposal. I first note that on some interpretations, quantum chances are modeled by ordinary 'classical' (Kolmogorovian) probability theory, not quantum probability theory, and so there is no need for a new treatment of the QPP on these interpretations. On other interpretations, Earman is right to point out that QPP requires its own special formulation, and that this is an unaddressed issue in the literature. However, I argue, Earman's proposed formulation is not the correct one. I lay out what I take to be the correct formulation, and show that although intuitively true, it does not follow as a theorem.

Before proceeding, it is worth mentioning a further reason that philosophers and physicists may be interested in the status of the QPP. An important practice within physics is the reconstruction of unknown quantum states through measurement. This process of quantum state tomography is one of several methods used to learn and estimate quantum states (Paris and Rehacek, 2004). To the extent we think of the physicists in these scenarios as rational agents with credences, we may be interested in modeling their learning experiences as they hone in on the unknown state. Here QPP plays a crucial role. Suppose $\psi$ is the true state of the system and $E$ is some experimental data. By Bayes' formula:

$$
C(\text { state is } \psi \mid E)=\frac{C(E \mid \text { state is } \psi) \cdot C(\text { state is } \psi)}{C(E)} \text {. }
$$

Crucially, the QPP fixes $C(E \mid$ state is $\psi)$ to $\psi(E)$. This ensures that the higher the chance $\psi$ assigns to the observation $E$, the stronger the true hypothesis is supported. The QPP also helps 
fix the value of the prior term $C(E)$ : letting $\left\{\psi_{i}\right\}$ denote the set of candidate states, we have

$$
C(E)=\sum_{i} C\left(E \mid \text { state is } \psi_{i}\right) \cdot C\left(\text { state is } \psi_{i}\right)=\sum_{i} \psi_{i}(E) \cdot C\left(\text { state is } \psi_{i}\right)
$$

in other words the agent's credence in the experimental outcome $E$ is equal to her expectation of its chance. The overall result is that the agent's credence in the state hypotheses evolves in the way familiar from Bayesian statistical inference (cf. Lewis (1980, pp. 285-7)). One of my criticisms will be that Earman's formulation of the QPP can't do the normative work required for this application to quantum state tomography, and statistical inference more generally. At the end of the paper, I will show that my formulation can.

\section{Earman on quantum chance}

In quantum probability theory, ${ }^{1}$ the objects of quantum chance - what I shall call, for lack of a better term, the quantum propositions - correspond mathematically to the set of all projection operators on the Hilbert space of the system in question. Quantum chance functions are then defined as functions on this set of quantum propositions. They output numbers between 0 and 1, and obey similar axioms to classical (Kolmogorovian) probability functions.

In more detail, let $\mathcal{H}$ be a separable Hilbert space and let $\mathfrak{N}$ denote the von Neumann algebra of all bounded operators acting on $\mathcal{H}$. A projection $E \in \mathfrak{N}$ is a self-adjoint element satisfying $E^{2}=E$. Let $\mathcal{P}(\mathfrak{N})$ denote the set of projections on $\mathfrak{N}$. Then a quantum chance function is a map $\omega: \mathcal{P}(\mathfrak{N}) \rightarrow[0,1]$ satisfying:

1. $\omega(I)=1$, where $I \in \mathcal{P}(\mathfrak{N})$ is the identity operator (analogue of the necessity), and

2. $\omega\left(\bigvee_{i} E_{i}\right)=\sum_{i} \omega\left(E_{i}\right)$ for any countable collection $\left\{E_{i}\right\}$ of mutually orthogonal projections. ${ }^{2}$

Example 1. In the Stern Gerlach case, we can consider the projection $P_{\uparrow_{x}}=\left|\uparrow_{x}\right\rangle\left\langle\uparrow_{x}\right|$ corresponding to the proposition that the 'UP' outcome will occur. Since the particle is in state $\uparrow_{z}$, the quantum

\footnotetext{
${ }^{1}$ Here I follow the presentation of Earman (2018); cf. also von Neumann (1955), Bub (1977), Hughes (1989, Ch. 8), Pitowsky (2006), Rédei and Summers (2007), Strocchi (2008, Ch. 2.4), and Hemmo and Shenker (2020).

${ }^{2}$ Here $\vee$ is the 'join' operator, the analogue of disjunction: if $E_{1}$ and $E_{2}$ are projection operators, then $E_{1} \vee E_{2}$ is the projection corresponding to the closure of $\operatorname{Ran}\left(E_{1}\right) \cup \operatorname{Ran}\left(E_{2}\right)$. Two projection operators are $E_{1}$ and $E_{2}$ are orthogonal when $E_{1} E_{2}=\mathbf{0}$. A collection is mutually orthogonal if every pair is orthogonal.
} 
chance function is given by $\omega(E)=\left\langle\uparrow_{z}|E| \uparrow_{z}\right\rangle$ for all projections $E \in \mathcal{P}(\mathfrak{N})$. One can verify that $\omega$ satisfies both axioms. In addition, $\omega\left(P_{\uparrow_{x}}\right)=\left\langle\uparrow_{z}\left|\left(\left|\uparrow_{x}\right\rangle\left\langle\uparrow_{x}\right|\right)\right| \uparrow_{z}\right\rangle=\left|\left\langle\uparrow_{z} \mid \uparrow_{x}\right\rangle\right|^{2}=1 / 2$ as expected.

We just saw that specifying the quantum state fixes the quantum chance function. It turns out that when the dimension of $\mathcal{H}$ is greater than two, the converse also holds: specifying the quantum chance function $\omega$ uniquely fixes a quantum state $\omega: \mathfrak{N} \rightarrow \mathbb{C}$, which we also denote $\omega .^{3}$ If $\omega$ is a vector state, in other words if there exists a vector $\psi \in \mathcal{H}$ such that $\omega(A)=\langle\psi|A| \psi\rangle$ for all $A \in \mathfrak{N}$, then we simply write $\omega$ as $\psi$.

What about credence functions? Earman assumes that in the quantum setting, rational initial credence functions $C$ are also defined over the quantum propositions $\mathcal{P}(\mathfrak{N})$. Furthermore, Earman imposes two basic coherence requirements on these functions:

Probabilism (quantum analogue): rational $C$ satisfy the axioms listed above (and hence can be extended to a state $C: \mathfrak{N} \rightarrow \mathbb{C}$, which we also denote $C$ ), and

Conditionalization (quantum analogue): rational $C$ obey an analogue of Bayesian updating, called Lüder's updating, which specifies that for all $E, F \in \mathcal{P}(\mathfrak{N}):^{4}$

$$
C(F \mid E)=\frac{C(E F E)}{C(E)}, \quad \text { provided } C(E)>0
$$

Say an initial credence function $C: \mathcal{P}(\mathfrak{N}) \rightarrow[0,1]$ is coherent if it satisfies these quantum analogues of probabilism and conditionalization. Earman's main claim is that any coherent credence function must automatically satisfy the QPP, thanks to the following result:

Theorem 1 (Ruetsche and Earman (2011), Fact 1). Set-up as above, where we suppose $\operatorname{dim}(\mathcal{H})>2$. Let $C: \mathcal{P}(\mathfrak{N}) \rightarrow[0,1]$ be any coherent initial credence function. Let $\psi \in \mathcal{H}$ be any vector state and let $P_{\psi} \in \mathcal{P}(\mathfrak{N})$ denote the projection onto the vector. ${ }^{5}$ Then, provided $C\left(P_{\psi}\right)>0$,

$$
C\left(\cdot \mid P_{\psi}\right)=\psi(\cdot) .
$$

\footnotetext{
${ }^{3}$ This follows from Gleason's theorem. Here a quantum state is a normed positive linear functional $\omega: \mathfrak{N} \rightarrow \mathbb{C}$ on $\mathfrak{N}$. Assuming $\omega$ is normal (cf. Ruetsche (2011)), there exists a unique density operator $\rho_{\omega} \in \mathfrak{N}$ with $\omega(A)=\operatorname{Tr}\left[A \rho_{\omega}\right]$ for all $A \in \mathfrak{N}$. In this case it is common to use $\omega$ and $\rho_{\omega}$ interchangeably.

${ }^{4}$ Here it is important that $C$ is extended to a state $C: \mathfrak{N} \rightarrow \mathbb{C}$, since for $F \in \mathcal{P}(\mathfrak{N})$ that do not commute with $E$, $E F E$ may not be a projection operator, thus leaving the numerator $C(E F E)$ undefined otherwise.

${ }^{5}$ Here it crucial that $\psi$ is a vector state, in particular that it is normal (cf. footnote 3) and pure; in ordinary QM, where $\mathfrak{N}$ is the von Neumann algebra, the pure normal states coincide with the vector states. Notably, the result cannot be extended to entangled $\omega$, for instance; see Section 4 for discussion.
} 
In other words, conditional on $P_{\psi}$, the credences $C$ must align with the chances $\psi$.

Based on this result, Earman concludes that the truth of the QPP is not a matter "to be submitted to the intuitions of wise analytical metaphysicians" but rather one to be proved: "credence and chance have been brought into alignment without the cudgel of any extra normative principle" (Earman, 2018, p. 10).

\section{Examining Earman's argument}

Note that for Earman's result to establish QPP, it is crucial that the projection $P_{\psi}$ represent the proposition $\langle$ the state of the system is $\psi\rangle$, or $\langle$ the chances are given by $\psi\rangle$. My main criticism of Earman's argument is that this claim is false, and so Equation (3) does not establish the Principal Principle.

Before I elaborate on this criticism, I should clarify an important issue regarding the scope of Earman's argument. Earman's choice to model quantum chances using quantum probability theory - and in particular to define chances over the set $\mathcal{P}(\mathfrak{N})$ of all projection operators - is not uncontroversial. Some quantum theories end up privileging a certain subset of commuting operators. This often brings them into the setting of ordinary 'classical' Kolmogorovian probability theory (Loewer, 1994). For example, in GRW theory, position is given a privileged role. The objective chances in this theory are the chances attached to spontaneous collapses of the position wave function. At a given time, the possible states of the world at the next time form the relevant set of possibilities. Chance is more naturally represented by a classical probability measure over this set: it selects which of these disjoint possibilities obtain, with the specific probabilities determined by the current spatial wave function and the given collapse constant. (Of course, spin and other degrees of freedom are still subject to chancy jumps, but only insofar as they are entangled with the spatial wave function.)

Similar remarks apply to Bohmian and Everettian mechanics. Bohmian mechanics is deterministic and so does not involve objective chances in Lewis's original sense. But insofar as it does feature a kind of objective chance, it is represented by an ordinary classical measure over initial Bohmian particle configurations. Again, this is related to the privileging of position in the theory. Similarly, the status of chance in the Everettian mechanics is a fraught issue, but to the extent 
it features in the theory, objective chance is more naturally represented as a classical probability measure, this time over disjoint branching possibilities. What makes this possible is the selection through decoherence of a preferred 'quasi-classical' basis over which branching occurs. (For a detailed treatment of both chance and Bayesianism in this setting, see Wallace (2012, Part II).)

The upshot is that on many quantum theories, standard treatments of the Principal Principle will carry over, and Earman's discussion won't apply. ${ }^{6}$ That said, on other interpretations, including but not limited to quantum logic interpretations (Stairs, 1983), information-theoretic interpretations (Bub, 2005), and certain pragmatist interpretations (Healey, 2017), quantum chances are understood as ranging over all projection operators. In these settings and others, Earman is correct to point out that we need to resituate QPP within quantum probability theory. Thus, for the rest of this paper, I will assume we are in such a setting.

With these caveats in mind, let us return to the criticism. In order for Earman's result (3) to establish the Principal Principle, it is crucial that $P_{\psi}$ represent the proposition $/$ the state of the system is $\psi\rangle$. But there is strong reason to reject this premise. For example, consider a spin$1 / 2$ particle whose state is entirely unknown. Then the proposition $\left\langle\right.$ the state is either $\uparrow_{z}$ or $\left.\downarrow_{z}\right\rangle$ is contingent, since it is possible that the true initial state is neither $\uparrow_{z}$ nor $\downarrow_{z}$ but rather some superposition of the two. However, $P_{\uparrow_{z}} \vee P_{\downarrow z}$ is a necessity; it coincides with the identity operator. More generally, for any orthonormal basis $\left\{\psi_{i}\right\}$, even though $\bigvee_{i}$ 〈the state is $\left.\psi_{i}\right\rangle$ may be contingent because the true state may be a superposition of the basis elements, $\bigvee_{i} P_{\psi_{i}}$ is always a necessity. This suggests that the projections $P_{\psi_{i}}$ do not represent propositions like $\left\langle\right.$ the state is $\left.\psi_{i}\right\rangle$, since they do not obey the same logic. To press the point, note that if $\psi_{1}$ and $\psi_{2}$ are distinct, we would expect $C\left(\right.$ state is $\psi_{1}$ or $\left.\psi_{2}\right)=C\left(\right.$ state is $\left.\psi_{1}\right)+C\left(\right.$ state is $\left.\psi_{2}\right)$, since either the true initial state is $\psi_{1}$, or it is $\psi_{2}$, or it is neither. However, it is well known (Strocchi, 2008, p. 51) that we may have

$$
C\left(P_{\psi_{1}} \vee P_{\psi_{2}}\right)>C\left(P_{\psi_{1}}\right)+C\left(P_{\psi_{2}}\right)
$$

when $\psi_{1}$ and $\psi_{2}$ are non-orthogonal. ${ }^{7}$

\footnotetext{
${ }^{6}$ This is not to say that quantum probability theory is misguided, or that it doesn't play an important role in our understanding of quantum mechanics. Rather this is to say that on certain specific foundational approaches, ordinary 'classical' probability theory is ultimately the more appropriate means to represent quantum chances.

${ }^{7}$ Again consider a spin-1/2 particle (examples in higher dimensions can also be given) and let $C$ be the coherent credence function corresponding to the vector state $\uparrow_{z}$. Suppose $\psi_{1}=\uparrow_{x}$ and $\psi_{2}=\downarrow_{z}$. Then $P_{\psi_{1}} \vee P_{\psi_{2}}=I$ so $C\left(P_{\psi_{1}} \vee P_{\psi_{2}}\right)=1$, but $C\left(P_{\psi_{1}}\right)+C\left(P_{\psi_{2}}\right)=\left|\left\langle\uparrow_{z} \mid \uparrow_{z}\right\rangle\right|^{2}+\left|\left\langle\uparrow_{z} \mid \downarrow_{z}\right\rangle\right|^{2}=1 / 2+0<1$.
} 
In response, one might object that just because $\left\langle\right.$ the state is $\psi_{i}$ 〉 is represented by $P_{\psi_{i}}$ for all $i$, it does not follow that the disjunction $\left\langle\right.$ the state is $\psi_{1}$ or $\left.\psi_{2}\right\rangle$ must be represented by $P_{\psi_{1}} \vee P_{\psi_{2}}$, or more generally that $\bigvee_{i}\left\langle\right.$ the state is $\left.\psi_{i}\right\rangle$ must be represented by $\bigvee_{i} P_{\psi_{i}}$. In particular, this step assumes that the disjunction in the state hypothesis must be interpreted as the join operator ' $V$ ' on the projections (which yields the closure of the union of their ranges), and this assumption could be denied. Of course, this line of response immediately faces the question of what operation, if not join, we are supposed to take the 'or' to correspond to. At least at first blush, there is no clear alternative operation, or alternative way of representing these disjunctive state hypotheses, yet we surely want to say that agents can entertain and assign credences to them.

But there is a bigger obstacle for this line of response, which is that the problems with representing state hypotheses by projections $P_{\psi}$ extend beyond cases involving the join operator $\vee$. Imagine that the agent is not sure which of some collection of states $\left\{\psi_{i}\right\}$ the system is in, but she is certain the system is in one of them, so $\sum_{i} C\left(\right.$ state is $\left.\psi_{i}\right)=1$. Suppose for simplicity that the collection $\left\{\psi_{i}\right\}$ is finite and forms an orthonormal basis. Recalling the discussion of quantum state tomography from the introduction (see (2)) we would expect

$$
C(E)=\sum_{i} C\left(E \mid \text { state is } \psi_{i}\right) \cdot C\left(\text { state is } \psi_{i}\right)
$$

i.e. the agent's credence in $E$ equals her expectation taken over the mutually exclusive and exhaustive hypotheses about the state. However, the example below shows that we may have

$$
C(E)>\sum_{i} C\left(E \mid P_{\psi_{i}}\right) \cdot C\left(P_{\psi_{i}}\right)
$$

even though $\sum_{i} C\left(P_{\psi_{i}}\right)=1$ and the $\left\{\psi_{i}\right\}$ are an ON basis, and hence the $\left\{P_{\psi_{i}}\right\}$ are partitional. This is further evidence that the projections $P_{\psi_{i}}$ do not represent state hypotheses. (For another similar cautionary tale about the interpretation of projection operators, see also Weinstein (1996).)

Example 2. Consider a spin-1/2 particle (the example can be extended to higher dimensions) and let the collection $\left\{\psi_{i}\right\}$ consist of $\uparrow_{z}$ and $\downarrow_{z}$. Let $C$ be the coherent credence function corresponding to the vector state $\uparrow_{x}$; note that $\sum_{i} C\left(P_{\psi_{i}}\right)=C\left(P_{\uparrow_{z}}\right)+C\left(P_{\downarrow_{z}}\right)=1 / 2+1 / 2=1$, as required. Now 
let $E=P_{\uparrow_{x}}$. Then $C(E)=1$, but $C\left(E \mid P_{\uparrow_{z}}\right) \cdot C\left(P_{\uparrow_{z}}\right)+C\left(E \mid P_{\downarrow_{z}}\right) \cdot C\left(P_{\downarrow_{z}}\right)=1 / 2 \cdot 1 / 2+1 / 2 \cdot 1 / 2<1 .^{8}$

An important consequence of this last result is that Earman's alleged Principal Principle, even if interpreted as such, does not do the normative work that it is supposed to do. (3) and (5) imply:

$$
C(E) \neq \sum_{i} \psi_{i}(E) \cdot C\left(P_{\psi_{i}}\right)
$$

even though $\sum_{i} C\left(P_{\psi_{i}}\right)=1$. If we do interpret $P_{\psi_{i}}$ as $\left\langle\right.$ the state is $\left.\psi_{i}\right\rangle$, as Earman's formulation of QPP suggests, then we are led to conclude that:

$$
C(E) \neq \sum_{i} \psi_{i}(E) \cdot C\left(\text { state is } \psi_{i}\right)
$$

even though $\sum_{i} C$ (state is $\left.\psi_{i}\right)=1$. In other words, the agent's credences need not match her expectations of the chances. ${ }^{9}$ But as we saw in the discussion of quantum state tomography, this is supposed to be a crucial consequence of QPP.

\section{Preparation, projective measurement, and a classical analogue}

If Earman's theorem does not establish QPP, then what does it establish?

The theorem establishes that if the system was knowingly prepared in state $\psi$ by a projective measurement with outcome $P_{\psi}$, then rational credence must align with $\psi$. Indeed this is the gloss that Earman offers in several places (2018, p. 10, notation adapted):

The intended interpretation of this [result] should be obvious. Suppose that an agent whose initial credence function on $\mathcal{P}(\mathfrak{N})$ is $C$ learns that a Yes-No experiment for $P_{\psi}$ has been performed and that a Yes answer has been returned. Since she is rational she Lüders updates her credence function to $C\left(\cdot \mid P_{\psi}\right)$. On the objectivist interpretation of

\footnotetext{
${ }^{8}$ Here we apply $(3)$ to obtain $C\left(E \mid P_{\uparrow_{z}}\right)=\uparrow_{z}(E)=1 / 2$ and $C\left(E \mid P_{\downarrow_{z}}\right)=\downarrow_{z}(E)=1 / 2$. More generally, let $d=\operatorname{dim}(\mathcal{H})$ where $1<d<\infty$. Let $\left\{\psi_{i}\right\}$ be an orthonormal basis and define $\varphi=\sum_{i} \sqrt{\frac{1}{d}} \psi_{i}$. Set $C(\cdot)=\varphi(\cdot)$ and $E=P_{\varphi}$. Then $C(E)=1$ but $\sum_{i} \psi_{i}(E) \cdot C\left(P_{\psi_{i}}\right)=\frac{1}{d} \cdot \sum_{i} C\left(P_{\psi_{i}}\right)=\frac{1}{d}<1$.

${ }^{9}$ It is true that given $C$ it can always be expressed as $C(\cdot)=\sum_{i} \psi_{i}(\cdot) \cdot C\left(P_{\psi_{i}}\right)$ for some orthonormal $\left\{\psi_{i}\right\}$. However the issue was whether given any collection of mutually exclusive and exhaustive chance hypotheses, the agent's credences should equal her expectations over those chance hypotheses. At the very least, we would expect that if all the $\left\{\psi_{i}\right\}$ assign the same chance to $E$, then $C(E)$ will equal that chance. But if the proposition $\left\langle\right.$ state is $\left.\psi_{i}\right\rangle$ is represented by the projection $P_{\psi_{i}}$ then this claim also fails: note that in the previous example, $\uparrow_{z}(E)=\downarrow_{z}(E)=1 / 2$ yet $C(E) \neq 1 / 2$.
} 
quantum probabilities, the returning of a Yes answer implies that the normal pure state $\psi$ has been prepared, and, hence, that the objective chance of an event $E \in P$ is $\psi(E)$, which is the same as the agent's updated credence, $C\left(E \mid P_{\psi}\right)$.

Although interestingly related to QPP, this result is not sufficient for it. There are two main ways to see that it falls short.

First, note that many preparations do not involve projective measurements (Fröhlich and Schubnel, 2016). For example, I can prepare a crystal in its ground state $\psi$ by leaving it in a cold room. Yet, QPP still requires I conform my credences to $\psi$, even though no projective measurement took place. Furthermore, it seems that even if no preparation of the system has taken place at all, for instance if I going to measure some cosmic rays incident on my laboratory that nobody has interacted with, QPP should still require I conform my credence in accordance with the expected state. Yet in these cases Earman's result won’t apply.

Second, the result only applies to vector states $\psi \in \mathcal{H}$. But suppose that the system $S$ is entangled with another $S^{\prime}$. If the agent knows $S$ 's state, it seems she should still be required to set her credences in line with that state, but since the state won't be pure, the result won't apply. (Here Earman could respond that we should move to the composite system $\mathcal{H}_{c}=\mathcal{H} \otimes \mathcal{H}^{\prime}$ and consider pure states $\psi_{c} \in \mathcal{H}_{c}$ of it. But I am imagining the agent is only probing $S$, so there is no "Yes-No experiment for $P_{\psi_{c}}$ " being performed, only Yes-No experiments for propositions of the form $F \otimes I$, where $F \in \mathcal{P}(\mathfrak{B}(\mathcal{H}))$ and $I \in \mathcal{P}\left(\mathfrak{B}\left(\mathcal{H}^{\prime}\right)\right)$ the identity.)

In fact, attending to this restriction to pure states, we can see that an exactly analogous result also holds in classical probability theory. Some preliminaries: Let $(S, \mathcal{S})$ be a classical outcome space, with $S$ a set and $\mathcal{S}$ a sigma-algebra on $S$. Define a state $\mu$ as a probability measure on $(S, \mathcal{S})$. A state $\mu$ is pure if it cannot be expressed as a trivial convex combination of other states. One can check that in this classical context, a state is pure if and only if it is a point measure concentrated at some $s \in S$, which I will write as $\mu_{s}$. It follows the pure states $\mu_{s}$ are in one-to-one correspondence with the elements of $S$. Next, define a projection operator as a random variable $X: S \rightarrow \mathbb{R}$ that satisfies $X^{2}=X$. One can check that every projection can be expressed as an 
indicator $X=1_{J}$ for some $J \in \mathcal{S}$, in other words a $\{0,1\}$-valued function:

$$
1_{J}(s)=\mu_{s}(J)= \begin{cases}1 & \text { if } s \in J \\ 0 & \text { if } s \in S \backslash J\end{cases}
$$

For some pure state $\psi=\mu_{s}$ we can consider the projection $P_{\psi}=1_{\{s\}}$ which tests whether $s^{\prime} \in S$ belongs to $\{s\}$. Like in the quantum case, we also associate $P_{\psi}$ with the claim that a Yes-No experiment for $P_{\psi}$ has been performed, and that a Yes answer has been returned: $P_{\psi}=\left\{s^{\prime} \in S\right.$ : $\left.1_{\{s\}}\left(s^{\prime}\right)=1\right\}=\{s\}$. Now, with these preliminaries in hand, we can obtain a classical analogue of Theorem 1:

Theorem 2 (Classical analogue). Let $C: \mathcal{S} \rightarrow[0,1]$ be any coherent initial credence function. Let $\psi$ be any pure state and let $P_{\psi}$ denote the projection defined above. Then, provided $C\left(P_{\psi}\right)>0$,

$$
C\left(\cdot \mid P_{\psi}\right)=\psi(\cdot)
$$

For proof, recall we can write $\psi=\mu_{s}$ for some $s \in S$ and $P_{\psi}=\left\{s^{\prime} \in S: 1_{\{s\}}\left(s^{\prime}\right)=1\right\}=s$ where we abuse notation and write $\{s\}$ as $s$. Now if $C(s) \neq 0$, then for all $J \in \mathcal{S}$,

$$
C\left(J \mid P_{\psi}\right)=C(J \mid s)=\frac{C(J s)}{C(s)}= \begin{cases}1 & \text { if } s \in J, \\ 0 & \text { if } s \in S \backslash J\end{cases}
$$

and so $C(J \mid s)=\mu_{s}(J)=\psi(J)$ as desired.

Intuitively, what is going on here is that since $\psi$ is pure, $P_{\psi}$ is an atomic proposition that specifies the exact outcome. Conditionalizing on this outcome, one is forced to align one's credence with the post-experiment chance of the outcome, which is either 0 or 1 . And so, in this very restricted sense, "credence and chance have been brought into alignment without the cudgel of any normative principle."

Needless to say, while a notable result, this does not constitute a proof of the Principal Principle. And as I have suggested here, although its quantum analogue is far more interesting, it is not a proof of the quantum Principal Principle either.

This discussion also makes evident the reliance, in Earman's result, on the choice to focus on the 
von Neumann algebra $\mathfrak{N}=\mathfrak{B}(\mathcal{H})$, which has atoms. In some quantum field theoretic settings, such as in the setting of Type-III factor algebras, there are no atoms in the relevant algebra (Ruetsche and Earman, 2011). The prospect of extending Earman's result to such settings looks dim.

\section{Formulating the Quantum Principal Principle}

I now present my positive proposal for how to formulate the QPP within the framework of quantum probability theory. As we'll see, while the resulting principle is highly intuitive, it does not follow as a theorem.

We begin by dividing the agent's credence function $C$ into two components, $x$ and $\mu$. The first component, $x$, is what I previously denoted $C$ : it is a function on the set of projections $\mathcal{P}(\mathfrak{N})$ that, at minimum, satisfies the coherence requirements from Section 2. The second component, $\mu$, is the key ingredient that was missing from Earman's approach. It is a (classical Kolmogorovian) credence distribution over the set of possible initial quantum states of the system. Roughly speaking, whereas $x$ represents the agent's ignorance about experimental outcomes, $\mu$ represents her ignorance about the true state. The QPP then constrains how $x$ and $\mu$ relate. It implies, for instance, that the agent's credence $x(E)$ equals her expectation of the chance of $E$,

$$
x(E)=\sum_{i} \psi_{i}(E) \cdot \mu\left(\psi_{i}\right) \quad \forall E \in \mathcal{P}(\mathfrak{N})
$$

where the different state hypotheses are weighted by $\mu$, and I have assumed, for simplicity, that $\mu$ is a discrete distribution over finitely many vector states $\left\{\psi_{i}\right\}$. Even at this point, we can see that QPP is not a theorem of quantum probability theory, since there are many pairs $(x, \mu)$, with $x$ and $\mu$ both coherent, that violate (8). For instance, suppose $x$ corresponds to a vector state like $\uparrow_{z}$ and $\mu$ assigns probability less than 1 to $\uparrow_{z}$. Then $x\left(P_{\uparrow_{z}}\right)=1>\sum_{i} \psi_{i}\left(P_{\uparrow_{z}}\right) \cdot \mu\left(\psi_{i}\right)$. As I understand the $\mathrm{QPP}$, then, it is a norm that goes beyond usual coherence requirements. Let us now formulate this norm in full detail.

Fix a separable Hilbert space $\mathcal{H}$ and associated algebra of bounded operators $\mathfrak{N}$. Let $K$ denote the set of quantum states on $\mathfrak{N}$. This set comes naturally equipped with the weak* topology. Let $\sigma(K)$ denote the sigma-algebra on $K$ generated by this topology. Now we can consider Kol- 
mogorovian probability measures $\mu$ on $(K, \sigma(K))$. As in Section 2, we can also consider quantum probability functions $x$ on $\mathcal{P}(\mathfrak{N})$. A coherent credence function $C$ is then modeled as a pair $(x, \mu)$.

Example 3. In the case of a spin one-half particle, $\mu$ can be thought of as a probability density over the Bloch sphere of spin states, and $x$ a point in the sphere. If $(x, \mu)$ obeys QPP, then $x$ will be the 'weighted average' of the points supported by $\mu$.

We are interested in how a rational $C$ changes with new evidence. If the evidence is an experimental outcome $E \in \mathcal{P}(\mathfrak{N})$, then we know $x$ updates by Lüder's conditionalization,

$$
x_{E}(F)=\frac{x(E F E)}{x(E)} \quad \forall F \in \mathcal{P}(\mathfrak{N})
$$

However, to know how $C$ updates, we also need to know how $\mu$ updates: after learning data $E$, how should the agent's credences in hypotheses $H$ about the (initial) state of the state of the system change? A coherent agent obeys Bayes' formula, so we set:

$$
\mu_{E}(H)=\frac{x_{H}(E) \cdot \mu(H)}{x(E)} \quad \forall H \in \sigma(K)
$$

In the case $H=\{\psi\}$, this is just a more elaborate way of stating (1).

What determines the agent's credence $x_{H}(E)$ in data $E$ given $H$ ? Enter the QPP:

Quantum Principal Principle (QPP): If $C=(x, \mu)$ is a rational initial credence function, then

$$
x_{H}(E)=\int_{K} \omega(E) d \mu_{H}(\omega)
$$

for all experimental outcomes $E \in \mathcal{P}(\mathfrak{N})$ and state hypotheses $H \in \sigma(K)$ such that $\mu(H)>0$.

Equation (10) says the agent's credence in data $E$ given state hypothesis $H$ is the expectation of the chance of $E$, restricted to states in $H$. In particular, here $\mu_{H}$ is defined in the standard way

$$
\mu_{H}(\cdot)=\frac{\mu(\cdot \cap H)}{\mu(H)} \quad \text { provided } \mu(H)>0
$$

which, substituted into (10), yields $x_{H}(E)=\frac{1}{\mu(H)} \int_{H} \omega(E) d \mu(\omega)$. 
In the special case where $H$ is the trivial (necessary) state hypothesis $K$, we obtain the result that the agent's unconditional credence in $E$ is her expectation of the chance of $E$,

$$
x(E)=\int_{K} \omega(E) d \mu(\omega), \quad \forall E \in \mathcal{P}(\mathfrak{N}) .
$$

In mathematical terminology, $x$ is the barycenter for $\mu$ (Alfsen, 2012). Note that this reduces to (8) when $\mu$ is a discrete distribution over finitely many vector states.

In the special case where $H=\{\nu\}$ is a hypothesis about a specific state $\nu$ then, assuming $\mu(\nu)>0$, QPP yields:

$$
x_{\nu}(E)=\frac{\int_{\{\nu\}} \omega(E) d \mu(\omega)}{\mu(\nu)}=\frac{\nu(E) \cdot \mu(\nu)}{\mu(\nu)}=\nu(E),
$$

in other words the agent's credence in $E$ given that the quantum state is $\nu$ must match the chance of $E$ according to $\nu$. This is exactly what QPP is supposed to capture. (And unlike the formulation (3), it applies both when $\nu$ is a vector state $\psi$ and when $\nu$ is an entangled state.)

Substituting these results into the Bayes' formula (9), we obtain:

$$
\mu_{E}(\nu)=\frac{\nu(E) \cdot \mu(\nu)}{\int_{K} \omega(E) d \mu(\omega)}
$$

If $\mu$ is a discrete distribution over finitely many vector states $\left\{\psi_{i}\right\}$, then this becomes, $\mu_{E}(\psi)=$ $\frac{\psi(E) \cdot \mu(\psi)}{\sum_{i} \psi_{i}(E) \cdot \mu\left(\psi_{i}\right)}$, which is just a way of spelling out Equations (1) and (2).

Example 4. A physicist has two identically prepared electrons. She knows they are either in state $\uparrow_{x}, \uparrow_{y}$, or $\uparrow_{z}$, and is equally confident in these three hypotheses. She then measures the first electron in the spin- $z$ direction and obtains an 'UP' outcome. How should her confidences over the hypotheses about the (initial) spin state change? Here we have

$$
\mu\left(\uparrow_{x} \otimes \uparrow_{x}\right)=\mu\left(\uparrow_{y} \otimes \uparrow_{y}\right)=\mu\left(\uparrow_{z} \otimes \uparrow_{z}\right)=\frac{1}{3} .
$$

The data the agent received is represented by $E=P_{\uparrow_{z}} \otimes I$. Assuming she satisfies QPP, her 
posterior credence in the hypothesis $\uparrow_{z} \otimes \uparrow_{z}$ is determined by (13):

$$
\mu_{E}\left(\uparrow_{z} \otimes \uparrow_{z}\right)=\frac{\frac{1}{3} \cdot\left(\uparrow_{z} \otimes \uparrow_{z}\right)(E)}{\left.\frac{1}{3}\left[\left(\uparrow_{x} \otimes \uparrow_{x}\right)(E)+\left(\uparrow_{y} \otimes \uparrow_{y}\right)(E)+\left(\uparrow_{z} \otimes \uparrow_{z}\right)(E)\right)\right]}=\frac{\frac{1}{3} \cdot 1}{\frac{1}{3}\left[\frac{1}{2}+\frac{1}{2}+1\right]}=\frac{1}{2} .
$$

As expected, the agent's credence in the $\uparrow_{z}$ hypothesis increases. A similar calculation shows that, as expected, her credence in the $\uparrow_{x}$ and $\uparrow_{y}$ hypotheses decreases:

$$
\mu_{E}\left(\uparrow_{x} \otimes \uparrow_{x}\right)=\frac{\frac{1}{3} \cdot \frac{1}{2}}{\frac{1}{3}\left[\frac{1}{2}+\frac{1}{2}+1\right]}=\frac{1}{4}, \quad \mu_{E}\left(\uparrow_{y} \otimes \uparrow_{y}\right)=\frac{\frac{1}{3} \cdot \frac{1}{2}}{\frac{1}{3}\left[\frac{1}{2}+\frac{1}{2}+1\right]}=\frac{1}{4} .
$$

So the agent begins with credence $(x, \mu)$ and ends with credence $\left(x_{E}, \mu_{E}\right)$, where $x_{E}(\cdot)=x(E$. $E) / x(E)$ and $\mu_{E}$ is specified by the above three equations.

What if the agent then obtains a second piece of experimental evidence $F \in \mathcal{P}(\mathfrak{N})$, say, through measuring the spin of the second electron? Here we need to check that our prescription for credal change is consistent, in the sense that if $E$ and $F$ commute, then beginning with $(x, \mu)$ and learning $E F$ gives the same result as receiving $E$, updating to $\left(x_{E}, \mu_{E}\right)$, and then learning $F$. A complication arises related to the fact that QPP is formulated for initial credence functions. If the agent already has total evidence $E$, then we need a slight generalization of (10):

$$
x_{E H}(F)=\int_{K} \omega_{E}(F) d \mu_{E H}(\omega) \quad \forall E \in \mathcal{P}(\mathfrak{N}), H \in \sigma(K), F \in \mathcal{P}(\mathfrak{N}),
$$

where $\mu_{E H}$ is defined as the result of conditioning $\mu_{E}$ on $H$, or equivalently, $\mu_{H}$ on $E$. Now, one can check that receiving $E F$, and so shifting to $x_{E F}(\cdot)=\frac{x(F E \cdot E F)}{x(E F)}$ and $\mu_{E F}(\cdot)=\frac{x_{(\cdot)}(E F) \cdot \mu(\cdot)}{x(E F)}$, gives the same result as receiving $E$ and then $F$, and so shifting to $\left(x_{E}\right)_{F}(\cdot)$ and $\left(\mu_{E}\right)_{F}(\cdot) .{ }^{10}$

$$
\begin{aligned}
& { }^{10} \text { For the derivation, first note that we have }\left(x_{E}\right)_{F}(\cdot)=\frac{x_{F}(E \cdot E)}{x_{F}(E)}=\frac{x(F E \cdot E F)}{x(F E F)} \text { and: } \\
& \begin{aligned}
\left(\mu_{E}\right)_{F}(\cdot)= & \frac{x_{E(\cdot)}(F) \cdot \mu_{E}(\cdot)}{x_{E}(F)}=\frac{\int_{(\cdot)} \omega_{E}(F) d \mu_{E H}(\omega) \cdot \mu_{E}(\cdot)}{\frac{x(E F E)}{x(E)}}=\frac{\frac{\int_{(\cdot)} \omega_{E}(F) d \mu_{E}(\omega)}{\mu_{E}(\cdot)} \cdot \mu_{E}(\cdot)}{\frac{x(E F E)}{x(E)}} \\
= & \frac{\int_{(\cdot)} \frac{\omega(E F E)}{\omega(E)} \cdot \frac{x_{\omega}(E)}{x(E)} d \mu(\omega)}{\frac{x(E F E)}{x(E)}}=\frac{\int_{(\cdot)} \frac{\omega(E F E)}{\omega(E)} \cdot \omega(E) d \mu(\omega)}{x(E F E)}=\frac{\int_{(\cdot)} \omega(E F E) d \mu(\omega)}{x(E F E)}=\frac{x_{(\cdot)}(E F E) \cdot \mu(\cdot)}{x(E F E)},
\end{aligned}
\end{aligned}
$$

where here for second equality we apply (14), for the fifth we apply (12), and for the last equality we apply (10). Second, note that since $E$ and $F$ are commuting projections, we have $x(F E F)=x(E F)=x(E F E)$. 
Example 4 (continued). The agent then measures the second electron in the spin- $y$ direction and obtains a 'down' outcome, represented by $F=I \otimes P_{\downarrow_{y}}$. How should her credences over her three hypotheses about the state change now? Again using the QPP, in particular (13) but with $\mu_{E}$ instead of $\mu$ determining the weights, we have

$$
\mu_{E F}\left(\uparrow_{z} \otimes \uparrow_{z}\right)=\frac{\frac{1}{2} \cdot\left(\uparrow_{z} \otimes \uparrow_{z}\right)(F)}{\frac{1}{4}\left[\left(\uparrow_{x} \otimes \uparrow_{x}\right)(F)+\left(\uparrow_{y} \otimes \uparrow_{y}\right)(F)\right]+\frac{1}{2}\left(\uparrow_{z} \otimes \uparrow_{z}\right)(F)}=\frac{\frac{1}{2} \cdot \frac{1}{2}}{\frac{1}{4}\left[\frac{1}{2}+0\right]+\frac{1}{2} \cdot \frac{1}{2}}=\frac{2}{3}
$$

By similar reasoning, $\mu_{E F}\left(\uparrow_{x} \otimes \uparrow_{x}\right)=\frac{1}{3}$ and $\mu_{E F}\left(\uparrow_{y} \otimes \uparrow_{y}\right)=0$. As expected, the $\downarrow_{y}$ result ruled out the hypothesis that the electrons were prepared in the $\uparrow_{y}$ state, but did not change the relative likelihood of the $\uparrow_{z}$ and $\uparrow_{x}$ hypotheses.

This treatment can be extended to more complicated quantum state tomography set-ups involving more copies, as well as other state estimation scenarios.

\section{Conclusion}

How do quantum chances constrain credence? At least some interpretations of quantum mechanics demand that we recast the Principal Principle within the framework of quantum probability theory. Earman (2018) sought to do so, and came to a surprising conclusion: the principle follows as a theorem. I started down the same path as Earman, but came to a different conclusion. The Quantum Principal Principle, appropriately formulated, is not a theorem of quantum probability theory; its justification does not come so straightforwardly. However, I still found that bringing the Principal Principle into the domain of quantum probability theory is a worthwhile and overlooked project. Not only does it yield new insight into the principle itself, as Earman showed, it also allows us to bring the principle to bear on important applications, like the learning of quantum states.

\section{Acknowledgments}

Many thanks to Gordon Belot, John Earman, Adam Elga, Hans Halvorson, Laura Ruetsche, Jeremy Steeger, and participants of the 2020 Ruetsche/Belot workshop series for their extremely helpful feedback and suggestions on this paper. 


\section{References}

Alfsen, E. M. (2012). Compact convex sets and boundary integrals, Volume 57. Springer Science \& Business Media.

Bub, J. (1977). Von neumann's projection postulate as a probability conditionalization rule in quantum mechanics. Journal of Philosophical Logic 6(1), 381-390.

Bub, J. (2005). Quantum mechanics is about quantum information. Foundations of Physics 35(4), $541-560$.

Builes, D. and J. Spencer (ms). Why chance constrains credence. Manuscript.

Childers, T. (2012). Objectifying subjective probabilities: Dutch book arguments for principles of direct inference. In D. Dieks, W. Gonzalez, S. Hartmann, M. Stoltzner, and M. Weber (Eds.), Probabilities, Laws, and Structures. Springer.

Earman, J. (2018). The relation between credence and chance: Lewis' "Principal Principle" is a theorem of quantum probability theory. Manuscript. Available at: http://philsci-archive. pitt.edu/14822/.

Fröhlich, J. and B. Schubnel (2016). The preparation of states in quantum mechanics. Journal of Mathematical Physics 57(4), 042101.

Healey, R. (2017). The Quantum Revolution in Philosophy. Oxford University Press.

Hemmo, M. and O. Shenker (Eds.) (2020). Quantum, Probability, Logic: The Work and Influence of Itamar Pitowsky. Jerusalem Studies in Philosophy and History of Science. Springer International Publishing.

Hughes, R. I. (1989). The structure and interpretation of quantum mechanics. Harvard university press.

Lewis, D. K. (1980). A subjectivist's guide to objective chance. In R. C. Jeffrey (Ed.), Studies in Inductive Logic and Probability, pp. 83-132. University of California Press. 
Loewer, B. (1994). Probability and quantum theory. In P. Suppes and P. Humphreys (Eds.), Patrick Suppes: Scientific Philosopher: Volume 2. Philosophy of Physics, Theory Structure, and Measurement Theory, pp. 3-28. Springer Netherlands.

Paris, M. and J. Rehacek (2004). Quantum State Estimation. Lecture Notes in Physics. Springer Berlin Heidelberg.

Pettigrew, R. (2012). Accuracy, chance, and the Principal Principle. Philosophical Review 121(2), $241-275$.

Pettigrew, R. (2013). A new epistemic utility argument for the Principal Principle. Episteme 10(1), $19-35$.

Pitowsky, I. (2006). Quantum mechanics as a theory of probability. In Physical theory and its interpretation, pp. 213-240. Springer.

Rédei, M. and S. J. Summers (2007). Quantum probability theory. Studies in History and Philosophy of Science Part B: Studies in History and Philosophy of Modern Physics 38(2), 390-417.

Ruetsche, L. (2011). Why be normal? Studies in History and Philosophy of Science Part B: Studies in History and Philosophy of Modern Physics 42(2), 107-115.

Ruetsche, L. and J. Earman (2011). Interpreting probabilities in quantum field theory and quantum statistical mechanics. In S. Hartmann and C. Beisbart (Eds.), Probabilities in Physics. Oxford University Press.

Schwarz, W. (2014). Proving the Principal Principle. In A. Wilson (Ed.), Chance and Temporal Asymmetry. Oxford University Press.

Stairs, A. (1983). Quantum logic, realism, and value definiteness. Philosophy of Science 50(4), $578-602$.

Strevens, M. (1999). Objective probability as a guide to the world. Philosophical Studies 95(3), 243-275.

Strocchi, F. (2008). An introduction to the mathematical structure of quantum mechanics: a short course for mathematicians, Volume 28. World Scientific. 
von Neumann, J. (1955). Mathematical Foudations of Quantum Mechanics. Princeton University Press.

Wallace, D. (2012). The emergent multiverse: Quantum theory according to the Everett interpretation. Oxford University Press.

Ward, B. (2005). Projecting chances: A Humean vindication and justification of the Principal Principle. Philosophy of Science 72(1), 241-261.

Weinstein, S. (1996). Undermind. Synthese 106(2), 241-251. 